\title{
A model for the formation of the active region corona driven by magnetic flux emergence ${ }^{\star}$
}

\author{
F. Chen $(\text { 陈枫 })^{1}$, H. Peter $^{1}$, S. Bingert ${ }^{1}$, and M. C. M. Cheung ${ }^{2}$ \\ 1 Max Plank Institut für Sonnensystemforschung, Justus-von-Liebig-Weg 3, 37077 Göttingen, Germany \\ e-mail: chen@mps .mpg.de \\ 2 Lockheed Martin Solar and Astrophysics Laboratory, 3251 Hanover St, Palo Alto CA 94304, USA
}

Received 16 October 2013 / Accepted 21 February 2014

\begin{abstract}
Aims. We present the first model that couples the formation of the corona of a solar active region to a model of the emergence of a sunspot pair. This allows us to study when, where, and why active region loops form, and how they evolve.

Methods. We use a 3D radiation magnetohydrodynamics (MHD) simulation of the emergence of an active region through the upper convection zone and the photosphere as a lower boundary for a 3D MHD coronal model. The coronal model accounts for the braiding of the magnetic fieldlines, which induces currents in the corona to heat up the plasma. We synthesize the coronal emission for a direct comparison to observations. Starting with a basically field-free atmosphere we follow the filling of the corona with magnetic field and plasma.

Results. Numerous individually identifiable hot coronal loops form, and reach temperatures well above $1 \mathrm{MK}$ with densities comparable to observations. The footpoints of these loops are found where small patches of magnetic flux concentrations move into the sunspots. The loop formation is triggered by an increase in upward-directed Poynting flux at their footpoints in the photosphere. In the synthesized extreme ultraviolet (EUV) emission these loops develop within a few minutes. The first EUV loop appears as a thin tube, then rises and expands significantly in the horizontal direction. Later, the spatially inhomogeneous heat input leads to a fragmented system of multiple loops or strands in a growing envelope.
\end{abstract}

Key words. Sun: corona - Sun: activity - Sun: UV radiation - sunspots - Sun: magnetic fields - magnetohydrodynamics (MHD)

\section{Introduction}

In the process of emergence of magnetic field through the surface of the Sun a group of sunspots can form and the upper atmosphere is heated and filled with plasma. This forms coronal loops prominently visible in extreme ultraviolet (EUV) and $\mathrm{X}$-ray observations. In the early phase of theoretical investigations of coronal loops, emphasis was put on their properties in an equilibrium state, i.e., once they have fully developed. For example, this led to the widely used scaling laws of Rosner et al. (1978) relating energy input and loop length to temperature and pressure of a static loop. While now time-dependent models including plasma flows are widely used, they mostly concentrate on the loop evolution in a magnetic field that changes only slightly or not at all (see, e.g., review by Reale 2010). This applies to 1D models for the dynamic evolution of loops, for example, accounting for siphon flows (Boris \& Mariska 1982), in response to intermittent nanoflare heating (Hansteen 1993), or concerning catastrophic cooling (Müller et al. 2003). In addition, 3D models of the coronal loop structure considered either a constant or slowly changing magnetic field in the photosphere (Gudiksen \& Nordlund 2002; Bingert \& Peter 2011; Lionello et al. 2013). In all these models, the loops form and evolve in a preexisting coronal magnetic field above a well developed active region. However, during the early formation stage of an active region, when the first coronal loops form, flux emergence is still significant. Using a magneto-frictional approach, Cheung \& DeRosa (2012) followed the whole evolution of an active region.

\footnotetext{
Animation associated with Fig. 2 is available in electronic form at http://www . aanda.org
}

In particular, they determined the coronal magnetic field in response to the flux emergence and used proxies based on currents to obtain a rough estimate of the coronal emission. The goal of our study is to go one step further and follow the formation of the first loops in an emerging active region in a self-consistent model, properly accounting for the plasma properties and the magnetic field. This requires the treatment of an energy equation including radiative losses, heat conduction, and plasma heating. The synthesized coronal emission can be directly compared to observations.

Over the last decade 3D models have shown that in the magnetically closed active region corona the heat input most probably is related to the tangling of the magnetic field in the photosphere, either through fieldline braiding by the horizontal motions of granulation (Parker 1972, 1983) or flux-tube tectonics (Priest et al. 2002). In both cases currents are induced in the corona and are subsequently dissipated. In a numerical experiment Galsgaard \& Nordlund (1996) could show that the driving of the magnetic field from the photospheric boundaries can lead to significant build-up of currents to heat the corona through Ohmic dissipation. In a more realistic setup Gudiksen \& Nordlund $(2002$, 2005a,b) showed that a million K hot loopdominated corona forms above an active region. They used an observed magnetogram and the fieldline braiding was driven by artificial granulation-like motions at their bottom boundary. The induced currents $j$ are then converted to heat through Ohmic dissipation $H_{\mathrm{Ohm}} \propto \eta j^{2}$, where $\eta$ has a functional dependence on $j$. Transition region EUV emission lines synthesized from these models match major observed features such as the transition region redshifts (Peter et al. 2004, 2006) or temporal variability 
(Peter 2007). Using a constant value for $\eta$, Bingert \& Peter (2011) studied the temporal variability of the heat input in detail. These models show that the energy is predominantly deposited in quantities of about $10^{17} \mathrm{~J}$ (Bingert \& Peter 2013), consistent with the predictions of nanoflares by Parker (1988). Recently, observed coronal structures could be reproduced by the 3D coronal models based on the observed magnetic field and flows in the photosphere alone (Bourdin et al. 2013), and the constant cross section of loops was reproduced (Peter \& Bingert 2012). While the above models are all based on DC-type Ohmic heating, recently van Ballegooijen et al. (2011) studied the AC energy input by Alfvén wave turbulence in a coronal magnetic flux tube using a reduced magnetohydrodynamics (MHD) approach and found it sufficient to heat the hot coronal loops.

On smaller spatial scales, more representative for network or plage patches, Martínez-Sykora et al. (2008, 2009) studied the process of a twisted flux tube emerging from the convective layer into the lower corona. In these realistic models the radiative transfer in the lower atmosphere is treated as well as the heat conduction and radiation needed in the coronal part. Descriptions of the flux tube emergence into the corona on larger scales, more representative of an active region, had to omit the realistic description allowing for the synthesis of photospheric and coronal emission (e.g., Fan 2001; Abbett \& Fisher 2003; Manchester et al. 2004; Archontis et al. 2004; Magara 2006). Hurlburt et al. (2002) used a 2D axisymmetric magneto-convection sunspot model to construct the coronal magnetic field above. In a collection of $1 \mathrm{D}$ static models along the coronal fieldlines they then derived the EUV and soft X-ray emission and compared it to observations. This model is still quite idealized in the sense that the corona is described by a series of $1 \mathrm{D}$ equilibrium models along magnetic fieldlines, and the heat input is determined by a (freely chosen) fraction of the Poynting flux that is generated by the magneto-convection.

In recent years it has become possible to study the fine structure of sunspots (Heinemann et al. 2007) and simulate a whole sunspot and its surrounding granulation in 3D models from the convection zone to the photosphere (Rempel et al. 2009). This paved the road to investigate the emergence of a flux tube through the surface resulting in a sunspot pair that is at the heart of an active region (Cheung et al. 2010). These models are realistic in the sense that they include all the relevant physics, in particular the radiative transfer, in order to produce synthetic images from the simulation that closely resemble actual observations. A variant of this latter model (see Sect. 2.1) will provide the lower boundary for our model of the corona above an active region.

The goal of our study is to use a realistic model of the emergence of a sunspot pair in the photosphere as a lower boundary to drive a coronal model of the active region corona above. A single model encompassing a whole active region from the interior to the upper atmosphere is not yet possible. Solving both the radiative transfer in the lower atmosphere and the anisotropic heat conduction in the corona in a computational domain covering a full active region is too demanding in terms of computing resources. Instead, we couple our coronal model to the flux-emergence simulation by driving the coronal model at the bottom boundary with the results from the flux-emergence simulation in the (middle) photosphere.

The model setup is described in Sect. 2, before we present the appearance of the model corona in Sect. 3. A detailed analysis along the coronal loop that forms first during the flux emergence is given in Sect. 4 followed by a discussion of the threedimensional nature of the loop in Sect. 5. Finally we discuss the trigger of the coronal loop formation in Sect. 6.

\section{Model setup}

\subsection{Flux emergence simulation}

The flux-emergence simulation we use to determine the lower boundary of our coronal model is based on the numerical experiment by Cheung et al. (2010). Here we actually use a variant with a slightly larger computational domain and without imposed twist for the emerging flux tube (Rempel \& Cheung 2014). In that model, the fully compressible MHD equations are solved including radiative transfer and a realistic (tabulated) equation of state. The computational domain covers $147.5 \times$ $73.7 \times 16.4 \mathrm{Mm}^{3}$, where the optical depth of unity is found about 15.7 $\mathrm{Mm}$ above the bottom. A torus-shaped magnetic flux tube containing $1.7 \times 10^{22} \mathrm{Mx}$ is kinematically advected into the domain through the bottom. The flux tube is then buoyantly rising through the upper convection zone and breaks into photosphere.

In the early stage of the flux emergence simulation, the flux tube is still below the photosphere and the photosphere is basically field-free. While the flux tube continues to rise, first a lot of small-scale flux concentrations emerge on the photosphere. Later, larger flux-concentrations form through the coalescence of small magnetic flux patches. In this process the granular motions play an important role. In the later stage of the simulation, some $27 \mathrm{~h}$ after the start of the simulation, a pair of sunspots forms in the photosphere. The diameter of each spot is about $15 \mathrm{Mm}$. The magnetic field strength in the center of the spots exceeds $3000 \mathrm{G}$, which is compatible with that of a large active region on the Sun. The total duration of the simulation was about $30 \mathrm{~h}$.

We extract the quantities in the photosphere $(\tau=1)$ of the simulation and use them to drive the coronal model (Sect. 2.3).

\subsection{Coronal model}

To model the corona above the emerging active region we employ a 3D MHD simulation. Vertically this stretches from the photosphere into to the corona. Our model follows the investigations by Bingert \& Peter $(2011,2013)$. We solve the induction equation together with the mass, momentum, and energy balance. We account for heat conduction along the magnetic fieldlines (Spitzer 1962), radiative losses in the corona (Cook et al. 1989), and Ohmic heating, so that the coronal pressure is set self-consistently. This allows us to synthesize the coronal emission to be expected from the model domain. As proposed by Parker (1983), magnetic fieldline braiding by photospheric motions induces currents in the corona, which are converted to heat by Ohmic dissipation. As in Bingert \& Peter $(2011,2013)$, we use a constant resistivity $\eta$. We set $\eta$ by requiring the magnetic Reynolds number to be about unity using the grid spacing as the length scale. This implies that $\eta$ is $10^{10} \mathrm{~m}^{2} / \mathrm{s}$. To numerically solve the MHD equations we use the Pencil code (Brandenburg $\&$ Dobler 2002) $)^{1}$. Further details on the model can be found in Bingert \& Peter (2011).

The domain of the coronal model matches that of the flux emergence simulation in the horizontal direction, i.e., it spans $147.5 \times 73.7 \mathrm{Mm}^{2}$. This is resolved by $256 \times 128$ grid points, implying a $576 \mathrm{~km}$ equidistant grid spacing. In the vertical direction the domain spans $73 \mathrm{Mm}$ above the photosphere. In the vertical direction we implement a stretched grid with 256 points. The vertical grid spacing near the bottom is about $32 \mathrm{~km}$, (matching the flux-emergence model), and in the coronal part it is about $300 \mathrm{~km}$.

\footnotetext{
1 See also http://pencil - code.googlecode.com/
} 
The initial atmosphere is determined by a temperature stratification similar to that of the Sun, which increases from $5100 \mathrm{~K}$ in the photosphere to $1 \mathrm{MK}$ in the corona with a smooth transition at about $3 \mathrm{Mm}$ above the photosphere. The initial density is calculated from hydrostatic equilibrium, with the density at the bottom boundary matching the average density of the extracted layers from the flux emergence simulation. The initial magnetic field is a potential field extrapolated from the photospheric magnetogram derived from the flux emergence simulation (see Sect. 2.3).

The lateral boundaries are periodic and the top boundary is closed, with a zero temperature gradient. The bottom boundary is set to drive the coronal model by the flux emergence simulation (Sect. 2.3). Our previous models (e.g., Bingert \& Peter 2011, 2013; Bourdin et al. 2013) used an observed magnetic field and an observed or generated velocity field to prescribe the bottom boundary conditions, which was then driving the model. Here we impose the properties from the flux-emergence simulation in a similar fashion.

\subsection{Coupling of the flux emergence and coronal model}

To drive the coronal model by the separate flux-emergence simulation, we have to couple the two simulations. This is done by specifying the bottom boundary condition of the coronal model, viz. the ghost layers and the bottom layer of the physical domain. For this we extract the density, temperature, and magnetic field in four consecutive layers from the photosphere in the fluxemergence simulation at a height of the average level of unity optical depth. We use a nearest-neighbor method to spatially interpolate the values in the horizontal direction to match the grid of the coronal simulation. Unfortunately, a lot of the kinetic energy on the small scales ( $\approx 70 \%$ of the total kinetic energy) is lost during the mapping. The impact of this on the coronal structure is discussed later in Sect. 6. The vertical spacing of the coronal model at the bottom and the flux-emergence simulation is identical. We store these values for the boundary and ghost layers with a cadence of $25 \mathrm{~s}$ which is more than sufficient to follow the emergence of the small flux elements in-between the granulation. At each time step of the coronal model when updating the boundary conditions, we feed the extracted values into the boundary and ghost layers of the coronal model. In this process we apply a linear interpolation in time between the boundary data stored with $25 \mathrm{~s}$ cadence.

Because no significant amount of magnetic flux is found in the photosphere before about $21 \mathrm{~h}$, and the spots do not start to form before $24 \mathrm{~h}$ into the flux-emergence simulation, we start the coronal model at $21 \mathrm{~h}$ after the start of the flux-emergence simulation. This should give enough time before magnetic concentrations form which are strong enough to give rise to the formation of coronal loops. The driving at the bottom boundary is switched on gradually within the first few minutes after the start. We also apply a damping to the velocity in the first $15 \mathrm{~min}$ to dissipate shocks generated from the mismatch between the initial condition and the bottom boundary. The coronal model is evolved for half a solar hour, with a basic energy equation without energy input, loss, or conductive transport, in order to relax from the initial condition. Then anisotropic heat conduction, radiative losses through optically thin radiation, and Ohmic and viscous heating are switched on. The coronal model evolves for about 8 solar hours almost to the end of the flux-emergence simulation, until the simulated spots are fully formed.

The main goal of this study is to investigate the initial formation of a coronal loop in an emerging active region. Therefore, in this paper we concentrate on a time span of about $30 \mathrm{~min}$ after the first coronal loop becomes clearly visible in the synthesized EUV emission, which is about $25.5 \mathrm{~h}$ after the start of the fluxemergence simulation.

\section{Coronal loops appearing in an emerging active region}

\subsection{Magnetic expansion into the corona}

Driven by the magnetic flux emergence through the bottom boundary, the magnetic field expands into the corona. In Fig. 1 we show an overview of the evolution of the magnetic field in the coronal simulation over four hours. We select the fieldlines at an early stage of the simulation by random seeds in a small volume in the lower middle of the computational box and trace their evolution ${ }^{2}$. Thus the lines in the three lower panels of Fig. 1 show the same set of fieldlines and how they evolve in time.

At $23.0 \mathrm{~h}$, there are already lots of small-scale fluxconcentrations in the photosphere. Low-lying fieldlines connect these small elements (not shown in the figure). As discussed in Cheung et al. (2010), these small flux concentrations are part of the flux tube brought to the surface through the near-surface convection. Because of the large scale of the emerging flux tube, the large-scale magnetic connections in the figure (at $23 \mathrm{~h}$ ) show a bipolar pattern.

After two hours evolution at about $25.2 \mathrm{~h}$, more magnetic flux emerged through the photosphere and the small-scale fluxconcentrations begin to coalesce. Now the large-scale magnetic field concentrations start to become visible in the photosphere. This is also illustrated by the fieldlines whose footpoints are moving closer to each other now concentrating near the simulated spots. The magnetic field strength near the footpoints increases. At the same time, the fieldlines expand upward into the higher atmosphere.

After another two hours, around $27.2 \mathrm{~h}$, a pair of simulated spots, where the magnetic field strength is over $3000 \mathrm{G}$, has formed in the photosphere. Now at the end of the coalescence process the footpoints of the fieldlines are bundled closely together. The central part of the set of fieldlines continues to expand into the higher corona.

The evolution of the magnetic field at the bottom boundary of our coronal model follows that in the flux emergence simulation, of course, albeit at a reduced spatial resolution. While most of the fine structures are lost due to the lower resolution, the photospheric magnetic field still captures the formation of sunspots by coalescence of small (down to the resolvable scale) flux elements.

\subsection{Appearance of a coronal loop}

One of the key interests of this study is whether coronal loops will form during the active region formation. Here and in most of the cases in this paper the term coronal loop refers to a loop-like structure identifiable in (real or model-synthesized) EUV observations of the corona. Whenever we refer to the magnetic field that confines the plasma contributing to the EUV loop emission, we always use the term magnetic tube.

\footnotetext{
2 The algorithm for fieldline tracing used by VAPOR with the results shown in Fig. 1 is described at http://www. vapor.ucar.edu/docs/ vapor-renderer-guide/flow-tab-field-line-advection. It assumes that fieldlines are frozen in the plasma elements (i.e., infinite conductivity) everywhere and follows the motions of plasma elements. The influence of magnetic diffusivity is discussed in Sect. 4.
} 

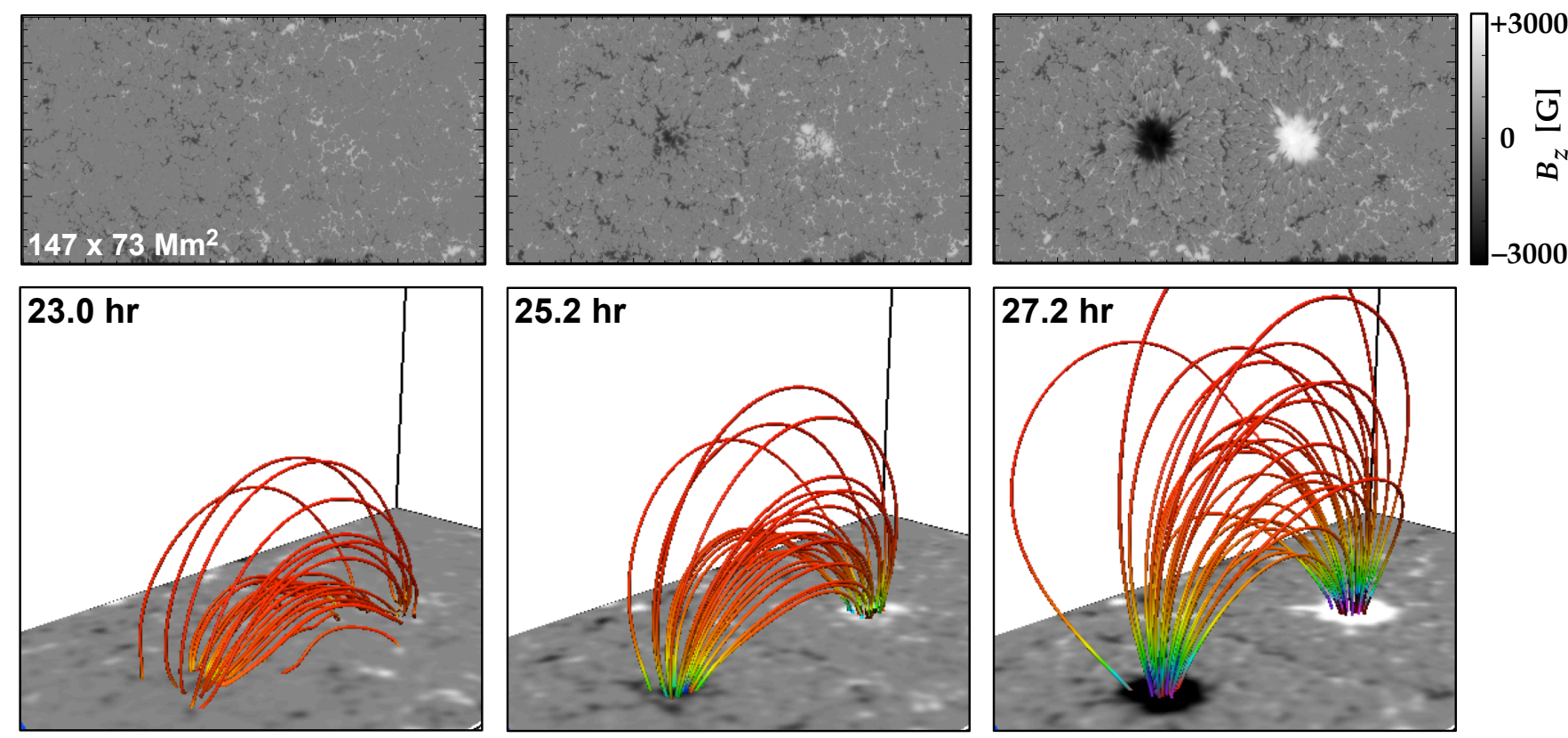

Fig. 1. Overview of the evolution of the magnetic field. The top row shows the magnetogram at the bottom boundary in the photosphere at three different times (vertical magnetic field). The horizontal extent in the top panels is $147.5 \times 73.7 \mathrm{Mm}^{2}$. The bottom row shows the evolution of a group of magnetic fieldlines in the coronal model driven by the flux emergence. The color coding on the fieldlines shows the magnetic field strength (red is low and purple is high). The bottom of the 3D rendering boxes are the same magnetogram as the top row. The times in the panels refer to the time since the start of the flux-emergence simulation. See Sect. 3.1.

To perform a direct comparison between our model and observations, we synthesize EUV images using the AIA response function (Boerner et al. 2012), following the procedure of Peter \& Bingert (2012). Here we concentrate at the AIA $193 \AA$ channel. It looks similar but not identical to $171 \AA$ and $211 \AA$ channels, which sample the 1 to 2 MK plasma, too. The $193 \AA$ and $211 \AA$ channels have also contributions from cooler plasma, in particular in quiet regions. However, this does not play a major role in our active region (model).

To form a coronal loop visible in EUV, one has to bring up enough plasma into the upper atmosphere along a fieldline and heat it to at least $10^{6} \mathrm{~K}$. In our model, the heating is by dissipation of currents which are induced by braiding of magnetic fieldlines through photospheric motions. If the magnetic field strength in the photosphere is low, the plasma motion can braid the magnetic fieldlines efficiently, but the induced currents will be weak, too. On the other hand, the braiding does not work in very strong magnetic flux concentrations, because there the plasma motions are suppressed. Both moderately high magnetic field and horizontal velocity are needed to induce enough current. This favorable combination is found at the periphery of sunspots, and in particular in our model after some $25.5 \mathrm{~h}$ after the start of the flux-emergence simulation. Therefore all times (usually given in minutes) mentioned in the remainder of the paper will refer to this time, i.e., in the following $t=0$ refers to $25.5 \mathrm{~h}$ after the start of the flux emergence simulation.

Once the energy input into the corona is sufficient, EUV loops will start to form. In Fig. 2 we show the synthesized images for the AIA $193 \AA$ channel integrated along the vertical and the horizontal coordinate at two different times 20 minutes apart. These views correspond to observations near the disk center (top panels) and at the limb (bottom panels). The left column shows a snapshot at $t=14 \mathrm{~min}$, just after the first EUV loop appears. At this time we see a single EUV loop forming, at later times more loops form at different places. Here we concentrate on the first single EUV loop in order to better isolate the processes triggering its formation ${ }^{3}$. The right panels of Fig. 2 show the coronal emission at $t=34 \mathrm{~min}$, after the loop started to fragment into several individual loops (see Sect. 5.2). From almost no emission to clearly detectable count rates in the synthesized images it takes only $\approx 5 \mathrm{~min}$ (see the animation with Fig. 2). In this paper we will mainly concentrate on the initial evolution of the loop system during about $15 \mathrm{~min}$.

The EUV loop is rooted in the periphery of the simulated spots, which is clear from the top panels of Fig. 2 showing an overlay of magnetogram and emission. This is consistent with the long-known observation fact that the footpoints of coronal loops are not in the umbra at the higher field strengths, but in the periphery, the penumbra (Bray et al. 1991). Even though the flux emergence simulation does not contain a proper penumbra (Cheung et al. 2010) it is clear that the loops are rooted in a region where convection can do considerable work to the magnetic field in the photosphere (see Sect. 6) and thus induces strong currents in the corona.

The visible top of the EUV loop rises upwards by $10 \mathrm{Mm}$ within $20 \mathrm{~min}$, which means a $10 \mathrm{~km} \mathrm{~s}^{-1}$ average upward velocity of the apex. The cross section of the EUV loop expands in the vertical direction during this rise. However, the EUV loop expands even more strongly in the horizontal direction after its initial appearance as a relative thin tube of up to $5 \mathrm{Mm}$ diameter and $45 \mathrm{Mm}$ length. Finally the emission covers the whole area in-between the two simulated spots, with a few fragments in the relatively diffuse loop emission (Fig. 2b).

The above discussion, in particular the late fragmentation, shows that a 3D treatment of the loop formation is essential. Still, in the early phase, the loop evolution appears to be close to a single monolithic loop. Therefore, we first analyze the $1 \mathrm{D}$ evolution

\footnotetext{
${ }^{3}$ In a more recent not yet fully finished numerical experiment we see also multiple loops forming at this early stage, so the limited spatial resolution in this model plays a role too.
} 

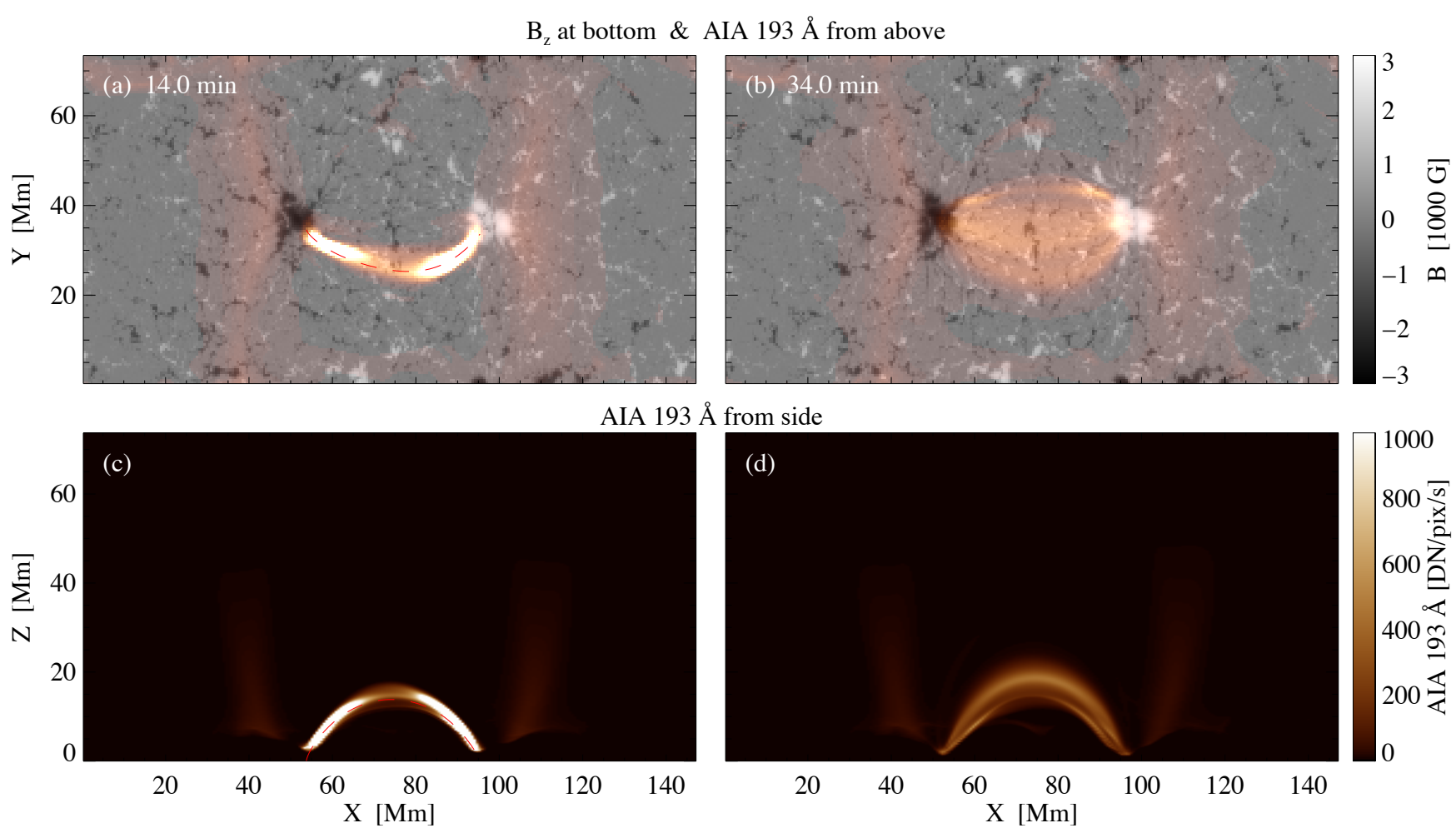

Fig. 2. Evolution of synthesized coronal emission and magnetic field. The top panels show photospheric magnetograms (vertical component), overlaid with the synthesized coronal emission as it would be seen in the AIA $193 \AA$ channel. The bottom panels show synthesized AIA $193 \AA$ images as seen from the side. The synthetic emission is integrated along the line of sight, comparable to what is seen at the disk center (top) or the limb (bottom). The two columns show snapshots from the simulation $20 \mathrm{~min}$ apart. Times refer to $25.5 \mathrm{~h}$ after the start of the flux-emergence simulation. The dashed line in the left panels shows the fieldline at the spine of the loop selected for the analysis in Sect. 4 . An animation of the figure is available online.

along the spine of the emerging loop in Sect. 4. The full 3D aspects and the trigger of the loop formation will be addressed after that in Sects. 5 and 6.

\section{The 3D loop collapsed to one dimension}

In the solar corona, the high electric conductivity prevents slippage of the fully ionized plasma across the field. The dominant magnetic energy assures that the Lorentz force determines motions perpendicular to the magnetic field. The anisotropic heat conduction quickly spreads the thermal energy along the fieldlines. Under such circumstances, often a simplified 1D model along a magnetic fieldline is sufficient to describe a coronal loop, although EUV loops are 3D structures in nature, as we show in this study.

In a $3 \mathrm{D}$ model, one can analyze the dynamics and thermal structures along a certain magnetic fieldline, which should then give results equivalent to that of a 1D loop model. For the loop forming in our 3D model, this applies in the early phase, when the loop is still confined to a thin magnetic tube. This assumption breaks down in the later phase, when the loop fragments into several substructures (see Sect. 5).

The spine of a EUV loop, which can be considered as being the central magnetic fieldline in the structure, is assumed to be static in most 1D models. However, it evolves in a selfconsistent manner in 3D models. For 3D models of mature active regions (Gudiksen \& Nordlund 2005a,b; Bingert \& Peter 2011), the magnetic field evolution follows the shuffling of footpoints of the fieldlines by granular motions and the change in morphology is very gentle. It is quite different in our model. When the first coronal loop becomes visible, the flux emergence is still going on, and the coronal magnetic field changes dramatically. To analyze the evolution of an equivalent 1D model, we need to follow the magnetic fieldline in time and extract all physical quantities along this evolving fieldline.

During this tracing, we assume that magnetic fieldlines are frozen-in with the plasma elements, as it should be in the case of high electric conductivity. Although there is a constant numerical resistivity in the induction equation in our simulation, the typical diffusion speed over $10 \mathrm{Mm}$ is on the order of $1 \mathrm{~km} \mathrm{~s}^{-1}$, which is smaller than the typical velocities (perpendicular to $B$ ) associated with the expansion of the magnetic fieldlines. In practice, we first follow the motion of the plasma element at the apex of a magnetic fieldline, and then calculate the new fieldline passing through the new position of this plasma element.

\subsection{Thermal structure and dynamics of the loop}

We choose a fieldline along the spine of the loop seen in the AIA $193 \AA$ image at $t=14$ min, when the loop is clearly defined (left panels of Fig. 2). In Fig. 3 we show a space-time diagram for this fieldline traced following the strategy above. The coordinate along the magnetic fieldline is normalized by the instantaneous length integrated between the two photospheric footpoints of the magnetic fieldline. During the time we investigate the evolution of the loop (approx. $14 \mathrm{~min}$ to $24 \mathrm{~min}$ ) the fieldline at its spine lengthens by some $10 \%$ to $15 \%$.

In the very early stage, the plasma along the fieldline is still cold and the pressure near the top is low. In this early phase 


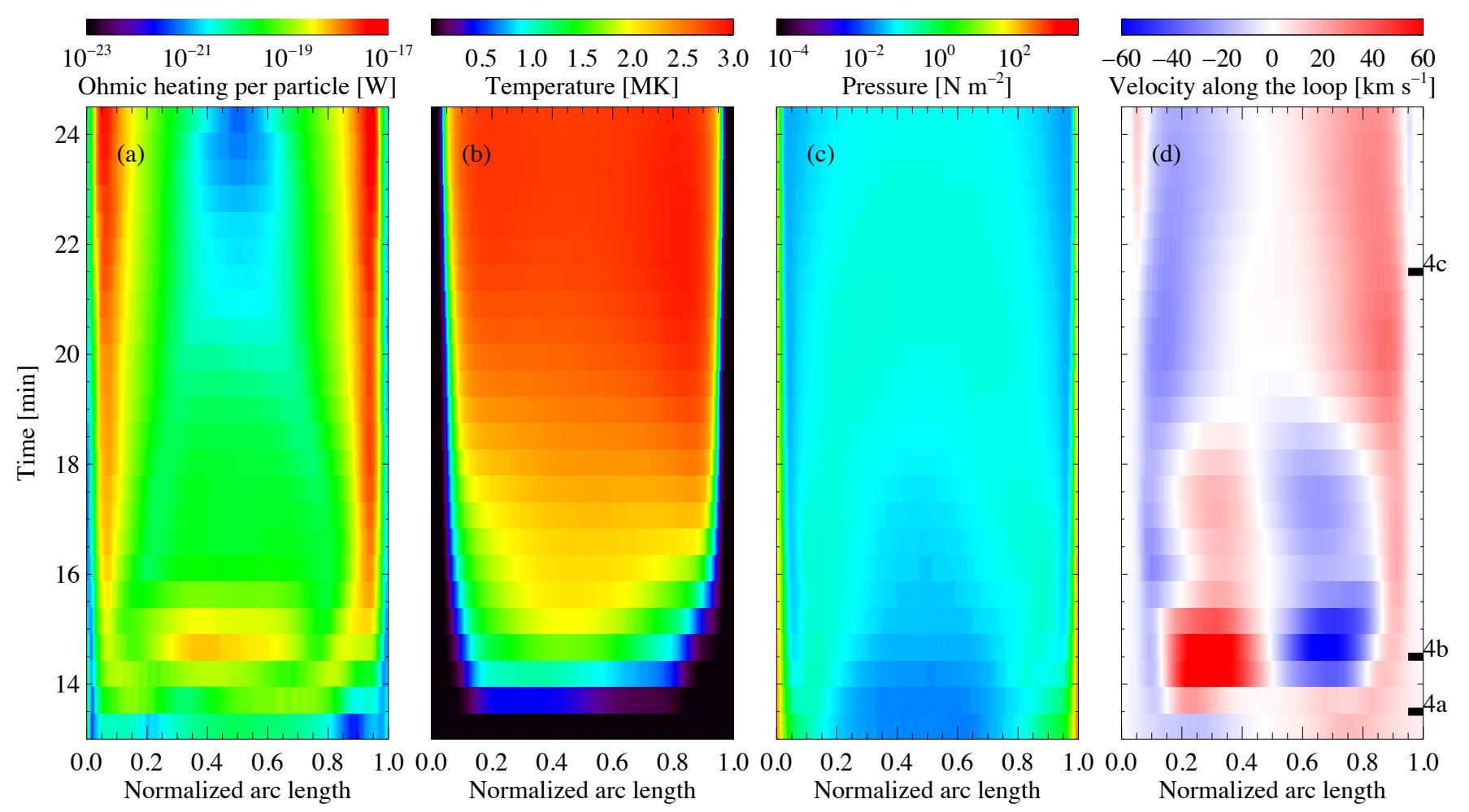

Fig. 3. Space-time diagram of emerging loop. All properties are shown as functions of normalized arc length along the fieldline at the spine of the forming loop and time. Because the fieldline is followed in time, its length is changing and the arc length along the fieldline is normalized to the length at the respective time. The loop footpoints are at arclengths 0 and 1 . In the velocity plot positive velocities (red) indicate a flow in the direction of arc lengths from 0 to 1 ("to the right"). In the color scale for the temperature green roughly corresponds to the peak contribution to the AIA $193 \AA$ channel. The marks 4a, 4b, and 4c in the right panel indicate the times shown in the three panels of Fig. 4. See Sect. 4.1.

$(t<14 \mathrm{~min})$, there is some weak draining along the fieldline, due to the slow rise of the fieldline driven by the flux emergence.

At $t \approx 14 \mathrm{~min}$, Ohmic heating increases through the whole loop, and the coronal temperature quickly increases to over $1 \mathrm{MK}$ (Fig. 3a, b). Here we analyze the temporal change in the heating, and discuss in Sect. 6 the self-consistent trigger of the increase of the heating rate in the 3D model. Very efficient heat conduction along the loop ensures an almost constant temperature along the fieldline in the coronal part (Fig. 3b). At the same time, the heat conduction transfers the energy deposited in the corona down to the cold dense chromosphere and induces an evaporation upflow (Fig. 3d). This flow fills the loop with plasma as is reflected by the increase of loop pressure in the Fig. 3c.

On the particular fieldline we analyze here, the plasma starts to increase its temperature at around $t=14 \mathrm{~min}$. On other fieldlines (reaching slightly greater apex heights) the heating sets in earlier. Thus some emission in the AIA $193 \AA$ channel can be seen already before $t=14 \mathrm{~min}$.

In a later stage $(t>16 \mathrm{~min})$, the Ohmic heating drops and the filling of the loop gradually ceases (Fig. 3a, d). The pressure gradient at this moment is not sufficient to balance gravity and thus to maintain an equilibrium. As a result, the plasma starts to drain, as demonstrated by the downflows in Fig. 3d after $t \approx$ $20 \mathrm{~min}$. The loop temperature, which is over $2.5 \mathrm{MK}$ after $t \approx$ $19 \mathrm{~min}$, is maintained for a long time. This is consistent with the long cooling times for these high temperatures (Klimchuk 2006), which is about $30 \mathrm{~min}$. The energy evolution of the loop is further analyzed in detail in Sect. 4.2.

There is a local pressure peak in the lower part on each side of the loop from $t=13.5 \mathrm{~min}$ to $18 \mathrm{~min}$. These peaks result in both upward and downward pressure gradient forces, which drive the flows to the loop top and the loop feet. The temperature of the downflow is $10^{4} \mathrm{~K}$ to $10^{5} \mathrm{~K}$, which corresponds to transition region temperatures; that of the upflow is $10^{6} \mathrm{~K}$, which corresponds to coronal temperature. This would cause the transition region lines (formed below $0.5 \mathrm{MK}$ ) to be redshifted and the coronal lines to be blueshifted. Thus, this is consistent with observations (Peter 1999; Peter \& Judge 1999) and in line with processes found by Spadaro et al. (2006) in 1D models and by Hansteen et al. (2010) in 3D models.

\subsection{Energetics in the emerging loop}

To investigate the energy budget controlling the thermal structure of the loop and its dynamics, we analyze the change in the thermal energy. We do this for the same magnetic fieldline chosen in Sect. 4.1. The conservation of thermal energy (see derivation in Appendix A) can be written as

$$
\begin{gathered}
\left(\frac{\partial e_{\mathrm{th}}}{\partial t}\right)_{s}=\underbrace{-u_{\|}(\mathbf{b} \cdot \nabla) e_{\mathrm{th}}}_{(1)}-\underbrace{-\frac{\gamma}{\gamma-1} p(\mathbf{b} \cdot \nabla) u_{\|}}_{(2)} \\
+\underbrace{Q}_{(3 \mathrm{a}, 3 \mathrm{~b})} \underbrace{-L}_{(4)} \underbrace{-\mathbf{b} \cdot \nabla q_{\|}}_{(5)} .
\end{gathered}
$$

Here $e_{\mathrm{th}}$ is the thermal energy per unit volume, following $e_{\mathrm{th}}=$ $p /(\gamma-1) . p$ is the plasma pressure, and $\mathbf{u}$ the velocity, $L$ denotes the radiative losses through optically thin radiation, $\mathbf{b}$ is the unit vector of the magnetic field, $u_{\|}=\mathbf{u} \cdot \mathbf{b}$ the velocity along the magnetic field, and $q_{\|}=-\kappa_{0} T^{5 / 2}(\mathbf{b} \cdot \nabla T)$ the heat flux along the magnetic field. Energy is added through $Q=Q_{\mathrm{Ohm}}+Q_{\text {visc }}$, with 

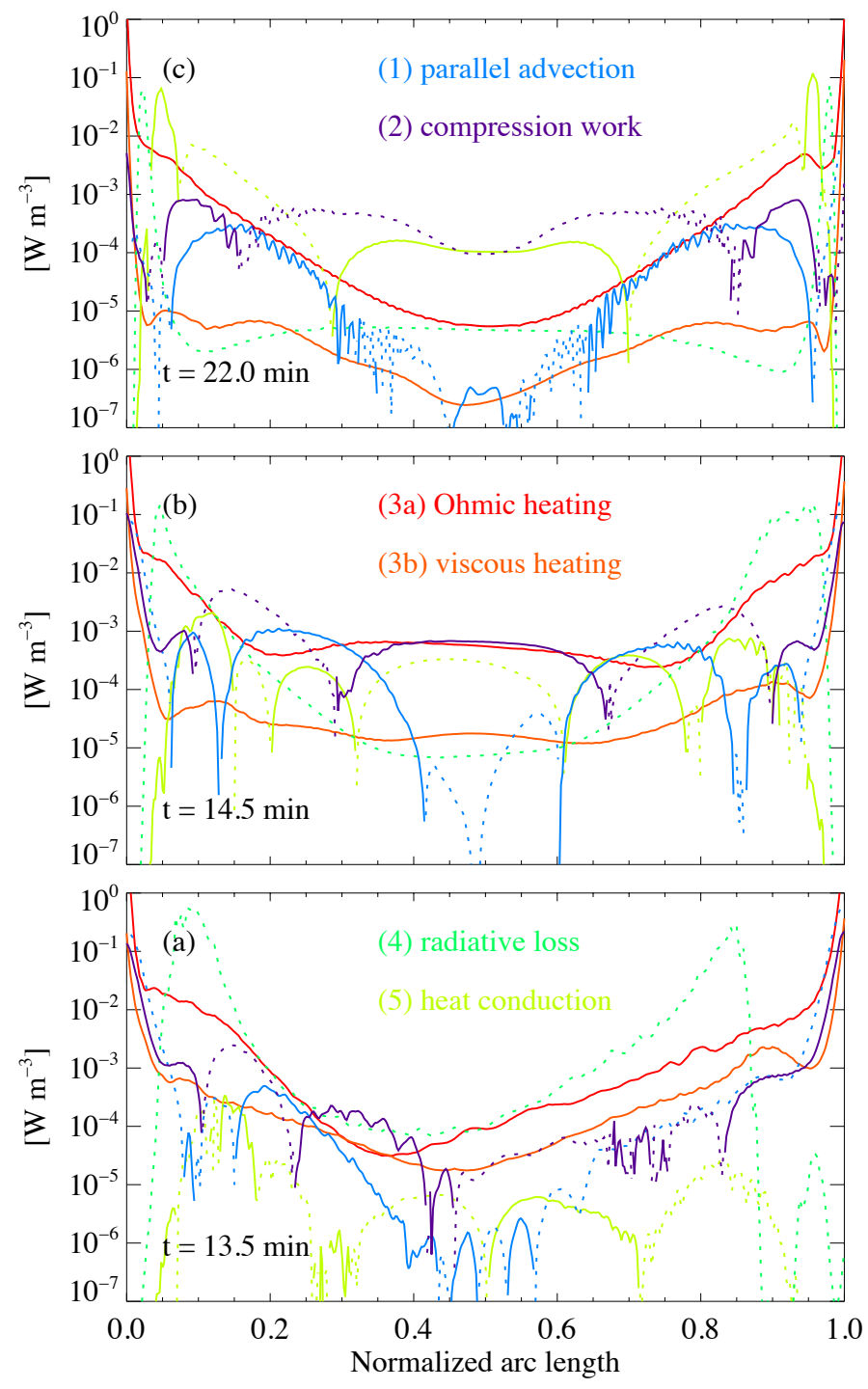

Fig. 4. Energy budget along the loop at three different times. The panels show snapshots during the phases of initiation a), formation $\mathbf{b}$ ), and cooling c) at the time given in each panel. These times are indicated to the right of Fig. $3 \mathrm{~d}$ by the marks $4 \mathrm{a}, 4 \mathrm{~b}$, and $4 \mathrm{c}$. The terms in Eq. (1) are shown accordingly to the labels in the plots, the numbers correspondingly to those in Eq. (1). The line colors follow the same definition in all panels. Dashed lines indicate negative, solid lines positive values. The arc lengths are normalized, with 0 and 1 corresponding to the two footpoints of the fieldline in the photosphere. See Sect. 4.2.

the Ohmic heating ( $3 \mathrm{a}) Q_{\mathrm{Ohm}}=\eta \mu_{0} j^{2}$ and viscous heating ( $3 \mathrm{~b}$ ) $Q_{\text {visc }}=2 \rho v \mathbf{S}^{2}$, where $\mathbf{j}$ is the current and $\mathbf{S}$ is the rate-of-strain tensor. In an equilibrium model, the time derivative and velocity would vanish, and the energy would be balanced between heating (3), radiative losses (4) and heat conduction (5). In our timedependent 3D model, the loop never reaches an equilibrium. The advection of thermal energy along the loop is given by term (1) and the compressional work (combined with the change in $e_{\text {th }}$ due to the compressibility of the plasma) by term (2) in the above equation.

In the following, we discuss the energy budget along the same fieldlines as in Sect. 4.1 before, during, and after the loop formation. For this we concentrate on the times $t=13.5 \mathrm{~min}$, $14.5 \mathrm{~min}$, and $22.0 \mathrm{~min}$ which are indicated in Fig. 3 by the marks $4 \mathrm{a}, 4 \mathrm{~b}$, and $4 \mathrm{c}$, which refer to the respective panels in Fig. 4 showing the terms (1) to (5) in the energy budget along the fieldline.

\subsubsection{Initiation phase}

In this early stage, there is a weak siphon flow in the loop (Fig. 3d), which is probably driven by the stronger heat input near the left footpoint. Because the loop is cool $\left(T<5 \times 10^{4} \mathrm{~K}\right)$, the heat input is more or less balanced by radiative losses (Fig. 4a). At this time the loop would be invisible in EUV images with count rates below the sensitivity (of AIA observations). But at this moment, the Ohmic heating starts to increase. Although at the normalized arc length of 0.3 viscous heating is of the same magnitude as Ohmic heating, it is in general lower than Ohmic heating by at least one order of magnitude. Thus the increase of Ohmic heating is the primary cause of loop formation.

\subsubsection{Formation phase}

At $t=14.5 \mathrm{~min}$, the Ohmic heating is high in the middle part of the fieldline, giving rise to the loop formation. Within less than a minute, the Ohmic heating rate has risen to a roughly constant level of almost $10^{-3} \mathrm{~W} \mathrm{~m}^{-3}$ in the hot coronal part (Fig. 4b). Considering that the coronal part covers $20 \mathrm{Mm}$ to $30 \mathrm{Mm}$ along the loop, this implies an energy flux of $\approx 10^{4} \mathrm{~W} \mathrm{~m}^{-2}$ into the loop, which would be consistent with estimates for coronal heating in active regions derived from observations (Withbroe \& Noyes 1977).

The Ohmic heating rate in other 3D MHD models (Gudiksen \& Nordlund 2005a,b; Bingert \& Peter 2011) drops (on average) exponentially with height, which is also true when following individual fieldlines (van Wettum et al. 2013). These previous models were describing a mature active region with a relatively stable magnetic configuration in which the footpoints are shuffled around. In contrast, in the present model the emerging magnetic field rises into the corona. Thus the interaction between the rising magnetic fieldlines hosting the loop and the ambient magnetic field also contributes to the currents along the loop, so that the Ohmic heating rate is quite constant along the loop (Fig. 4b). The viscous heating is almost two orders of magnitude lower, so that the Ohmic heating dominates the energy input.

The heat conduction term is negative near the apex, i.e., it transports the energy added by the Ohmic heating to the lower part of the loop. Ultimately, the energy is radiated close to the footpoints where the temperature is low.

The advection term at normalized arc lengths of 0.15 to 0.3 (and symmetric on the other side of loop) demonstrates the evaporation upflow filling the loop (Fig. 3d). This converging flow towards the loop top provides compressional work adding energy near the loop apex. This compressional work nearly equals the Ohmic heating at the loop top.

The effect of all contributions, i.e., the right-hand side of Eq. (1), is positive. This leads to a net rise of $\partial e_{\mathrm{th}} / \partial t$ on the order of $e_{\text {th }} / \tau \approx 10^{-3} \mathrm{~W} \mathrm{~m}^{-3}$ (see Fig. 4b). In the coronal part of the loop the number density is about $n \approx 10^{9} \mathrm{~cm}^{-3}$. Therefore the required increase of the energy $e_{\mathrm{th}}=\frac{3}{2} n k_{\mathrm{B}} T$ to reach coronal temperatures of about $T \approx 10^{6} \mathrm{~K}$ is on the order of $\tau \approx 1 \mathrm{~min}$. This time is compatible with the synthesized images, in which we see the loop forming in a matter of minutes (see animation attached to Fig. 2).

In their 2D study Hurlburt et al. (2002) implicitly assumed that the corona adjusts instantly to changes in the heat input 


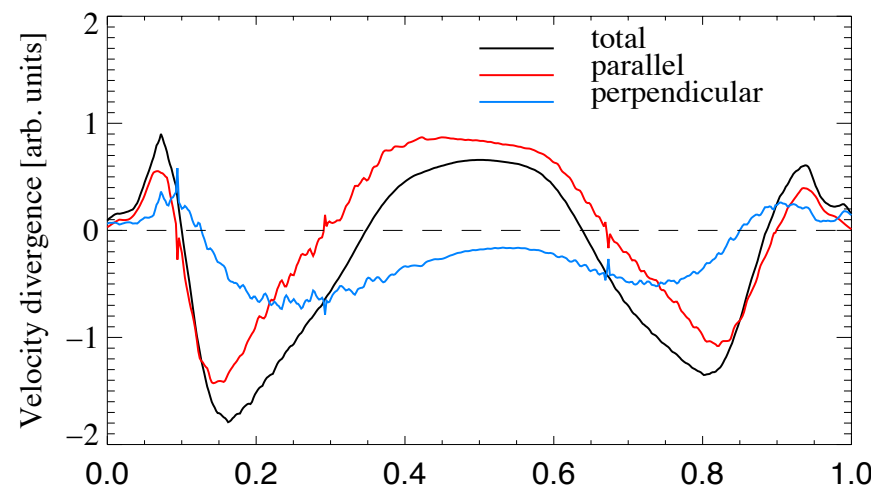

Fig. 5. Divergence of velocity along the loop at $t=14.5 \mathrm{~min}$ during the loop formation phase. The total divergence $-\nabla \cdot \mathbf{u}$ is shown in black, the parallel component in red, and the perpendicular component in blue. The arc length along the fieldline is normalized to the loop length. See Sect. 4.2.4.

because they employ a series of (static) equilibrium models. Here we see that the timescale for the evolution of the loop (minutes) is comparable to the timescale of the energy injection from the photosphere through the Poynting flux (see also Sect. 6). Thus one has to account for the evolution of the thermal properties in a dynamic model.

\subsubsection{Cooling phase}

After the heating ceases the loop enters a slow cooling phase (Fig. 4c). Owing to the drop of Ohmic heating, the plasma pressure falls, the plasma loses its support, and the loop drains. Consequently, decompression is the dominating cooling agent at the apex, as is illustrated by the negative contribution of the compression work throughout the top half of the loop (Fig. 4c). Along with the draining, advection transports energy from the loop top to the lower parts. In this late phase the dominant heating of the apex is due to heat conduction from the sides. Potentially, such situations can lead to a loss of equilibrium and catastrophic cooling (Müller et al. 2003, 2004), which we do not observe here because the heating is not concentrated strongly enough towards the footpoints.

In $3 \mathrm{D}$ models with a more stable magnetic field configuration, the loop can reach a (quasi-)equilibrium state, and remain stable for a longer time (Peter \& Bingert 2012). In our model, the magnetic field is expanding due to the flux emergence. Thus the loop cannot reach a (quasi-)equilibrium state and remains a transient feature evolving fast on a timescale of much less than 30 min (see animation with Fig. 2). This is underlined by the fact that the main cooling agent (in the top part) is decompression of the plasma driven by the expansion of the magnetic field.

\subsubsection{Perpendicular compression}

A static rigid 1D loop model only accounts for the compression work from velocity parallel to the magnetic fieldline. However, the compression or expansion perpendicular to the fieldline contributes to the thermal energy density in a 3D model, in particular, if the loop is expanding and interacting with the ambient magnetic field, as it is the case here.

To evaluate the role of the perpendicular compression, we split the divergence of the velocity, $\nabla \cdot \mathbf{u}$, into its parallel component $\left((\mathbf{b} \cdot \nabla) u_{\|}\right)$and its perpendicular component. The latter is evaluated by $\nabla \cdot \mathbf{u}-(\mathbf{b} \cdot \nabla) u_{\|}$. Figure 5 shows these contributions

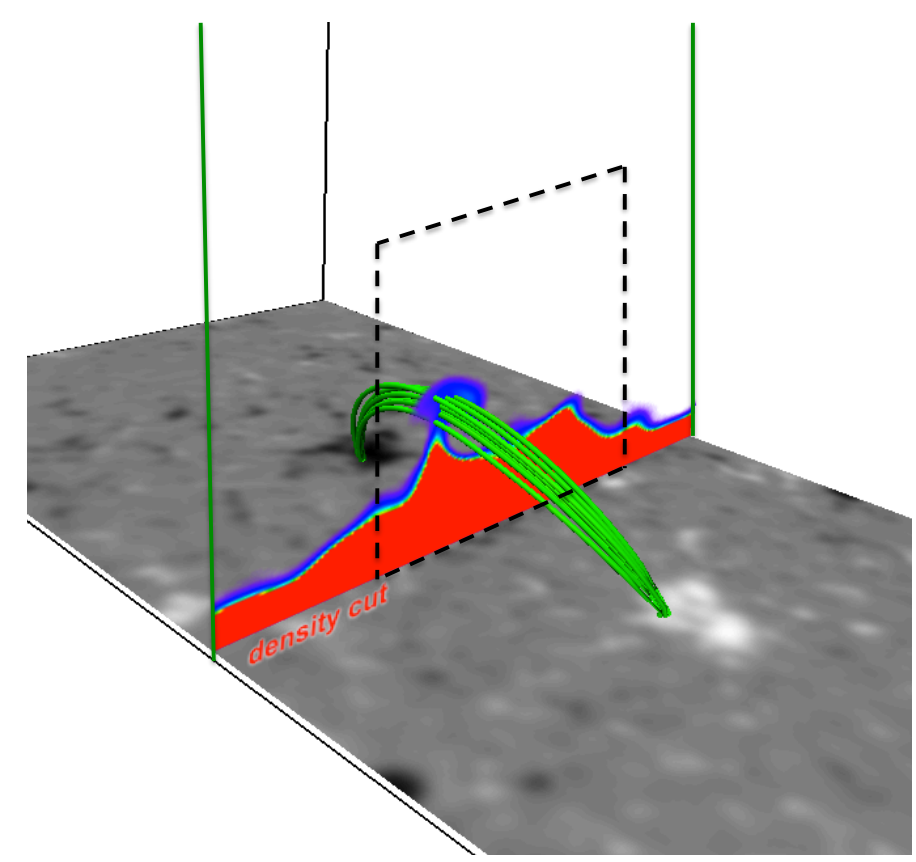

Fig. 6. 3D visualization of the location of the bright loop at time $t=14 \mathrm{~min}$. The green lines show the fieldlines roughly outlining the volume of the first bright loop appearing in the simulation. The bottom plane shows the vertical component of the photospheric magnetic field. A density cut in the vertical plane halfway between the footpoints of the loops perpendicular to the loop is indicated by the large green square. On this cut red indicates chromospheric densities, and blue enhanced coronal densities of about $10^{9} \mathrm{~cm}^{-3}$. Lower densities in the corona are transparent. The black square in dashed line on the midplane indicates the field of view in Fig. 8. See Sect. 5.

at $t=14.5 \mathrm{~min}$, i.e., during the loop formation phase. For consistency with Fig. 4 we plot $-\nabla \cdot \mathbf{u}$. A positive value in Fig. 5 implies convergence/compression, and a negative one implies divergence/expansion. Near the loop top, the parallel contribution shows a converging pattern, because evaporation flows from loop footpoints meet at the loop top (cf., Fig. 3). In contrast, the perpendicular contribution shows a diverging pattern at the top. This is corresponding to the expansion of the magnetic tube which will be discussed in Sect. 5.1. Still, the net effect is a compression of the plasma. In the lower part of the loop the total divergence is basically determined by the parallel contribution.

Although the perpendicular divergence has non-negligible contribution throughout the loop, the profile of the total divergence mostly follows that of the parallel contribution. This suggests that the $1 \mathrm{D}$ description of the flow in the loop is still acceptable at this stage. However, later the loop shows a clear $3 \mathrm{D}$ nature, which is discussed in the next section.

\section{The 3D nature of the loop}

\subsection{Evolution of the magnetic envelope}

To study the magnetic envelope of the EUV loop seen in the synthesized $193 \AA$ images, we investigate the evolution of a magnetic tube that is (at one particular time) roughly co-spatial with the volume of the EUV loop. We define the magnetic tube based on a vertical cut perpendicular to the loop plane in the middle between the two sunspots $(x=73 \mathrm{Mm})$ at the time $t=14 \mathrm{~min}$ (see Fig. 6). On this plane, we choose several points roughly enclosing the cross section of the synthesized AIA $193 \AA$ loop as 


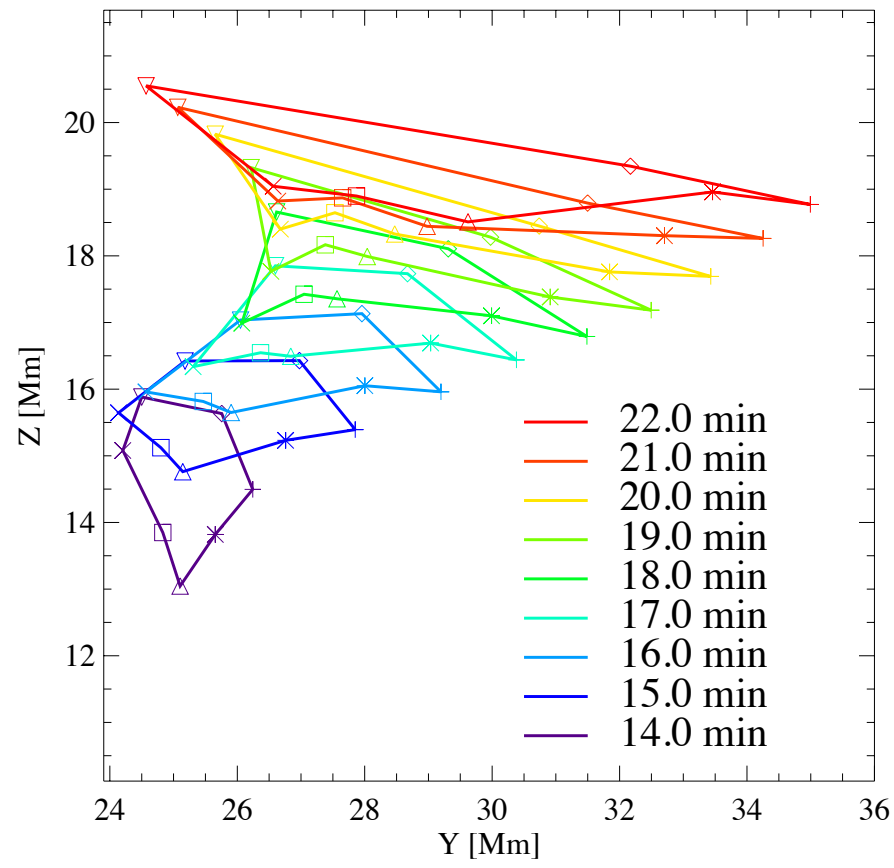

Fig. 7. Evolution of cross section of the magnetic tube roughly encompassing the loop appearing in the synthesized AIA $193 \AA$ images (cf. Fig. 2). The symbols show the positions of the fieldlines used to define the magnetic tube in the vertical midplane between the loop footpoints (cf. green square in Fig. 6). The same symbols indicate the same fieldline at the times color-coded according to the key. See Sect. 5.1.

starting points to follow magnetic fieldlines. This set of fieldlines defines the magnetic tube that we study further. We follow the magnetic tube in time by the same method as used in Sect. 4 and investigate the evolution of the cross section of the tube in the vertical midplane between the loop footpoints (large green square in Fig. 6).

We depict the temporal evolution of the cross section of the magnetic tube in the vertical midplane in Fig. 7. The magnetic tube moves upward as a whole and the cross section is significantly deformed. From $t=14 \mathrm{~min}$ to $t=22 \mathrm{~min}$ the cross section contracts in the vertical direction and expands significantly in the horizontal direction. This appearance of the magnetic tube is consistent with the rise of the synthesized AIA $193 \AA$ loop spine in the vertical direction and its significant horizontal expansion (see Fig. 2 and attached animation). An oblate shape of flux tubes was recently also reported by Malanushenko \& Schrijver (2013) who analyzed the cross section of thin flux tubes in a potential field model. They found that the cross section is distorted for the end-to-apex mapping. That the magnetic tubes in the corona will be non-circular in cross section has already been reported before (Gudiksen \& Nordlund 2005b).

\subsection{Fragmentation of the loop}

In Fig. 2 (and the attached animation) one can see that the synthesized AIA $193 \AA$ loop is a thin bright structure at the early stage, and then expands. The single bright loop breaks into several individual strands, which is best seen in the top view of the box (Fig. 2b). We use the term fragmentation for this process. Inspecting the temporal evolution in the animations attached to Fig. 2 it is clear that this fragmentation means that the original loop fades and new fragments or strand continuously form and
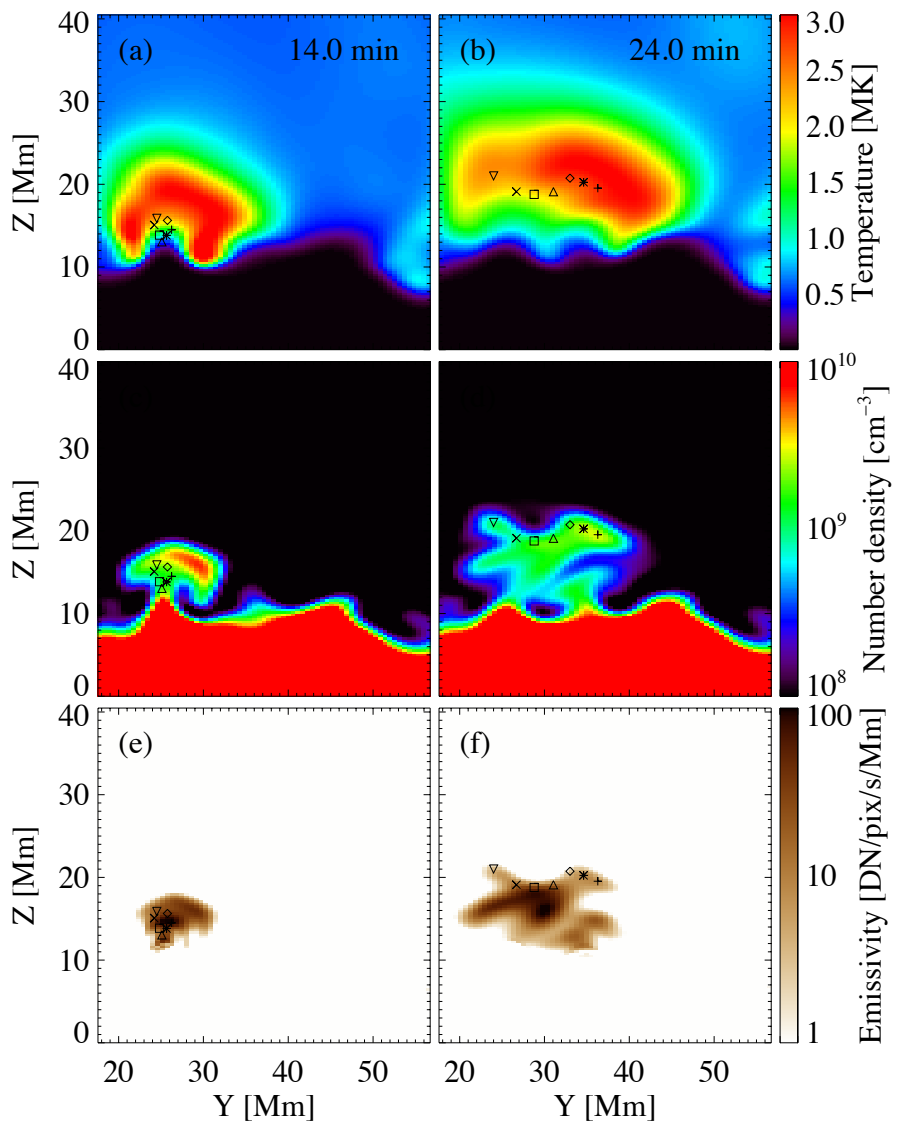

Fig. 8. Temperature, density and synthesized AIA $193 \AA$ emission in a vertical midplane between the loop footpoints (cut at $x=73 \mathrm{Mm}$ ). The left and right columns show snapshots $10 \mathrm{~min}$ apart at the times indicated in the top panels. In the temperature plots green roughly represents the temperature of maximum contribution to the $193 \AA$ A channel. The black symbols indicate the cross section of the magnetic tube discussed in Sect. 5.1 and Fig. 7. The field of view roughly matches the black square in dashed line in Fig. 6. See Sect. 5.2.

dissolve, giving the overall impression of a fragmentation. So this fragmentation is not to be understood in a way as a piece of wood would splinter, but as a coming and going of strands in a growing envelope.

To investigate this process, we show in Fig. 8 vertical cuts through the box in the midplane between the two footpoints. This midplane is roughly perpendicular to the loop (same plane as discussed above, cf. Fig. 6). The fragmentation of the loop is visible in the coronal emission emerging from this plane (bottom row of Fig. 8). At the later stage (Fig. 8f) individual patches of AIA $193 \AA$ emission have formed that would correspond to individual strands of the larger envelope.

To understand this EUV loop fragmentation we have to investigate the temperature and density structure in the vertical plane. For this we show in Fig. 8 also the temperature (top) and density (middle) in the vertical midplane. During the $10 \mathrm{~min}$ between the two snapshots shown in the left and right columns, the temperature and density structures move upward, and expand horizontally. The density structure looks less smooth than the temperature which is in part because of the draining and filling of the corona.

The $193 \AA$ emissivity (bottom row) is the product of the density squared and the temperature response function for that channel (Boerner et al. 2012). The latter largely (but not only) 
reflects the contribution function of FexII, which is strongly peaked with a maximum near 1.5 MK. In effect, the strong $193 \AA$ emission originates from locations where the density is high and the temperature is near the peak of the response function for this particular channel. Consequently, the $193 \AA$ emission pattern is neither cospatial with the density nor with the temperature structure, as is also clear from comparing the panels in the right column of Fig. 8 at the later time. The emission structure appears to be much more fragmented than both the temperature and density structure. This is simply because the density and temperature structures are not cospatial, and thus the convolution of the (smooth) density and temperature structures leads to the more clumpy coronal $193 \AA$ emission. The same is also true for the other AIA coronal channels, which we do not show here.

We note that we find in this work a temperature gradient perpendicular to the loop spine with an increasing temperature with height (from about $z=14 \mathrm{Mm}$ to $22 \mathrm{Mm}$ ). This is similar to the model of Peter \& Bingert (2012) who proposed a new mechanism to explain the constant cross section of coronal loops. Thus some parts of the high density structure at higher temperatures are cut off by the temperature response (or contribution) function, and in EUV emission the loops looks as if having a constant cross section, even though the plasma loop, i.e., the density structure, expands along the loop, or more precisely, with the magnetic tube. Even though we do not investigate this further in detail here, the $193 \AA$ loop shown in Fig. 2 from the side has roughly constant cross section. This is based on the same process as outlined by Peter \& Bingert (2012).

\section{What triggers the loop formation?}

The appearance of the model corona is compatible with EUV observations in the sense that a clearly distinguishable loop forms in the synthesized images. The question remains why the loop forms at that particular time and position. We investigate this by checking the energy input into the loop which is given through the Poynting flux, $\tilde{\mathbf{S}}=\left(\eta \mathbf{j}-\mathbf{u} \times \mathbf{B} / \mu_{0}\right) \times \mathbf{B}$. Near the bottom boundary, the driving by the photospheric convective motions from the flux-emergence model induces strong currents, which are mainly confined to the bottom layers. The amplitude of the resistive term, $\eta \mathbf{j}$ drops very fast with height and becomes much smaller than the $\mathbf{u} \times \mathbf{B} / \mu_{0}$ term, in particular in the area near the simulated spots. Thus when studying the energy input into the coronal part of the loop it is sufficient to investigate the $\mathbf{u} \times \mathbf{B}$ part alone,

$\mathbf{S}=-\frac{1}{\mu_{0}}(\mathbf{u} \times \mathbf{B}) \times \mathbf{B}$.

In Fig. 9 we show the vertical component of the Poynting flux $\mathbf{S}$ in horizontal slices at three heights, from the photosphere $(z=$ $0.32 \mathrm{Mm})$ to the coronal base $(z=2.02 \mathrm{Mm})$. There is a clear enhancement of the upward-directed Poynting flux surrounding the sunspot areas forming sort of a ring around the sunspot (green in Fig. 9 for $z=0.32 \mathrm{Mm}$ ). This enhancement is at least a factor of five to ten with respect to the surrounding quiet Sun area or the center of the sunspot. In the former the magnetic field is too weak, in the latter the strong magnetic field suppresses the horizontal motions, so that in these regions no considerable upward directed Poynting flux can be found. This is consistent with the widely known observational fact that coronal loops in EUV and X-rays do not originate from the center of sunspots where the magnetic field is the strongest, but from the periphery of sunspots, i.e., the outer parts of the penumbra. In our model
AIA $193 \AA$
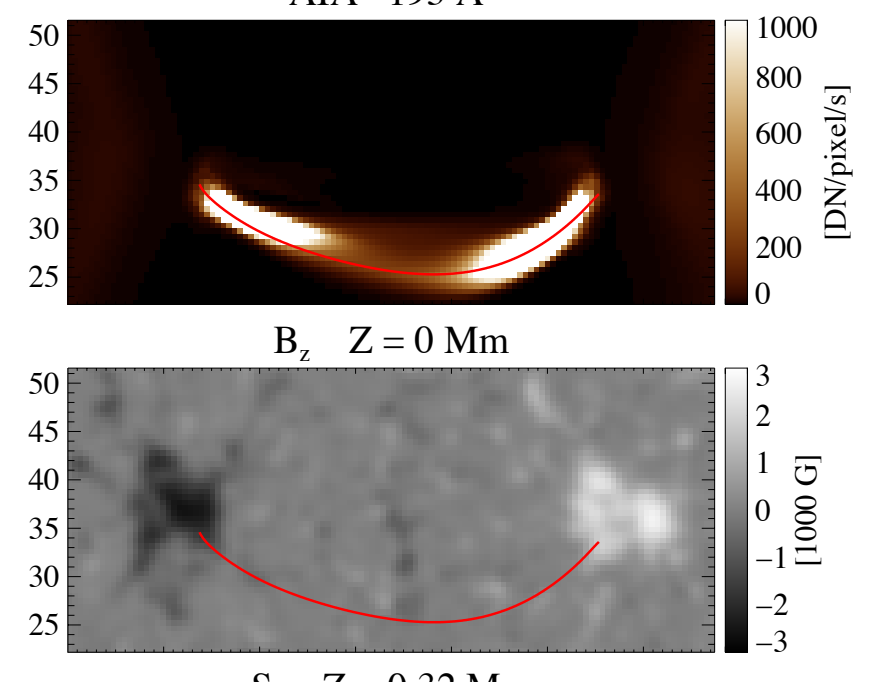

$\mathrm{S}_{\mathrm{z}} \quad \mathrm{Z}=0.32 \mathrm{Mm}$
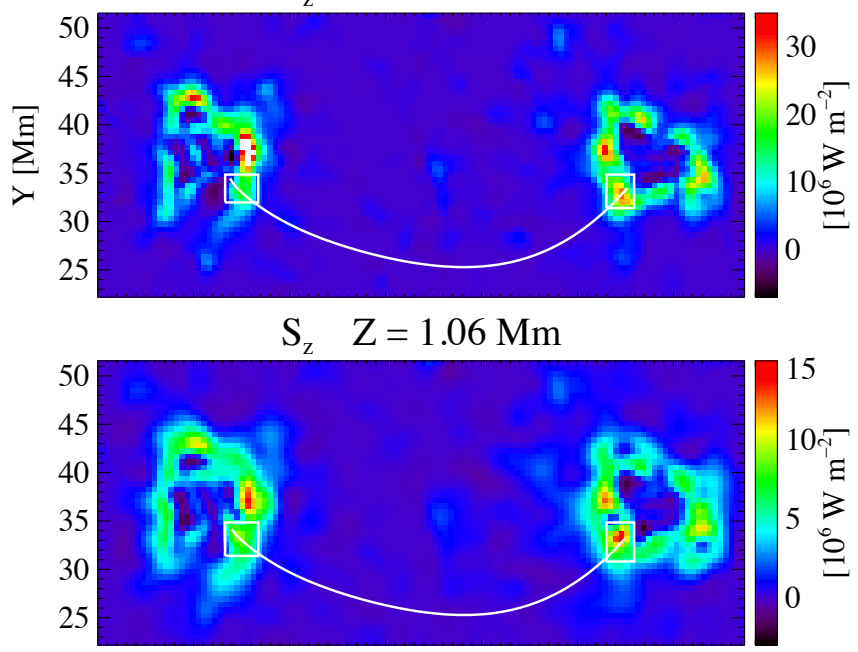

$\mathrm{S}_{\mathrm{z}} \quad \mathrm{Z}=2.02 \mathrm{Mm}$



Fig. 9. Zoom into the emerging active region at $t=14 \mathrm{~min}$. The top two panels show the synthesized AIA $193 \AA$ emission integrated along the vertical and the magnetic field, $B_{z}$, at the bottom boundary. The lower three panels show the vertical component of the Poynting flux, $S_{z}$, at three heights. The red and white lines indicate the same magnetic fieldline at the spine of the loop as shown in Figs. 2. The white boxes around both footpoints in the lower panels indicate the regions where we calculate the average vertical Poynting flux in Fig. 10.

this is reflected by the fact that only in the periphery is the upward Poynting flux significantly enough to power coronal loops.

At the coronal base $(z=2.02 \mathrm{Mm})$ the Poynting flux has the strongest enhancement near both footpoints of the loop, being typically another factor of about three higher than in the already 
enhanced region in the sunspot periphery. In Fig. 9, this shows up as the red spots in the panel for $z=2.02 \mathrm{Mm}$. However, in the photosphere $(z=0.32 \mathrm{Mm})$ only the right footpoint shows an enhancement of the Poynting flux, but not the left one. A closer inspection at the bottom boundary shows that this enhancement near the right footpoint in the photosphere is due to small magnetic flux elements which are advected by the convective motions into the strong magnetic field of the sunspot. These magnetic flux elements have sizes of $\approx 3 \mathrm{Mm}$, which is the scale of energy input into the loop and is not too far from the smallest resolvable scale in this model.

We miss a lot of the small-scale motions and fine magnetic structures in the photosphere when we map the original fluxemergence simulation to the grid of the coronal simulation (see Sect. 2.3). This can have two consequences. First, the energy input into the corona in our model is reduced, because we miss the Poynting flux on these smaller scales, at most this is a factor of two. Because the temperature scales with the energy input to the power of 2/7 (Rosner et al. 1978), this would have only a minor impact on the temperature, but it might be that the coronal density in our model is too low by up to a factor of 2 in some places. Second, the higher spatial resolution in the photosphere, properly resolving granulation, will give rise to finer structures in the corona, too. These conclusions are supported by the preliminary results from a high-resolution numerical experiment.

To further investigate the vertical Poynting flux at the footpoints of the loop we study the temporal variation of the vertical Poynting flux in different heights along the loop. We do this in terms of averages in a small horizontal section around the loop as indicated by the rectangles in Fig. 9. The sizes of the rectangles are slightly different for the left and right footpoints and for different heights in order to best capture the Poynting flux enhancement. The positions of the rectangles are fixed in time. The resulting averages as a function of time are plotted in Fig. 10.

At $z=0.32 \mathrm{Mm}$, the vertical Poynting flux at the right footpoint (solid) increases significantly by more than $6 \mathrm{MW} / \mathrm{m}^{2}$ during the $12 \mathrm{~min}$ shown in the plot around the time the loop appears (Fig. 10). A Fourier analysis clearly shows that this increase is modulated with a timescale of about $4 \mathrm{~min}$, which is close to the 5 min oscillations in the photosphere and close to the lifetime of granules. In contrast, the left footpoint shows no significant increase over time, but only the granulation modulation. That the left and the right footpoint show a different behavior in the photosphere is not surprising, because in the flux emergence simulation these two footpoint regions, which are located in the different sunspots, evolve independently.

The situation is different higher up in the atmosphere. At $z=1 \mathrm{Mm}$ and $2 \mathrm{Mm}$ both footpoints show a significant increase, with the right footpoint preceding the rise of the left footpoint at both heights.

Based on the timing shown in Fig. 10 one can sketch the following scenario. At the right footpoint in the low photosphere the upward Poynting flux is increasing because of the nearsurface convection driven by the flux emergence simulation. This disturbance of the field then travels upward through the highplasma- $\beta$ region and can be seen in the upper photosphere near $z=1 \mathrm{Mm}$ and further propagates upward to the coronal base at $z=2 \mathrm{Mm}$, where plasma- $\beta$ is below unity. Here we can see a steeper rise after the magnetic stresses have been built up slowly from below. We also see a clear rise on the left footpoint at the coronal base at $z=2 \mathrm{Mm}$. However, this increase lags behind the rise in the right footpoint by some $30 \mathrm{~s}$, which is close to the Alfvén crossing time (with a loop length above $z=2 \mathrm{Mm}$ of about $40 \mathrm{Mm}$ and an average Alfvén speed of about $2000 \mathrm{~km} \mathrm{~s}^{-1}$

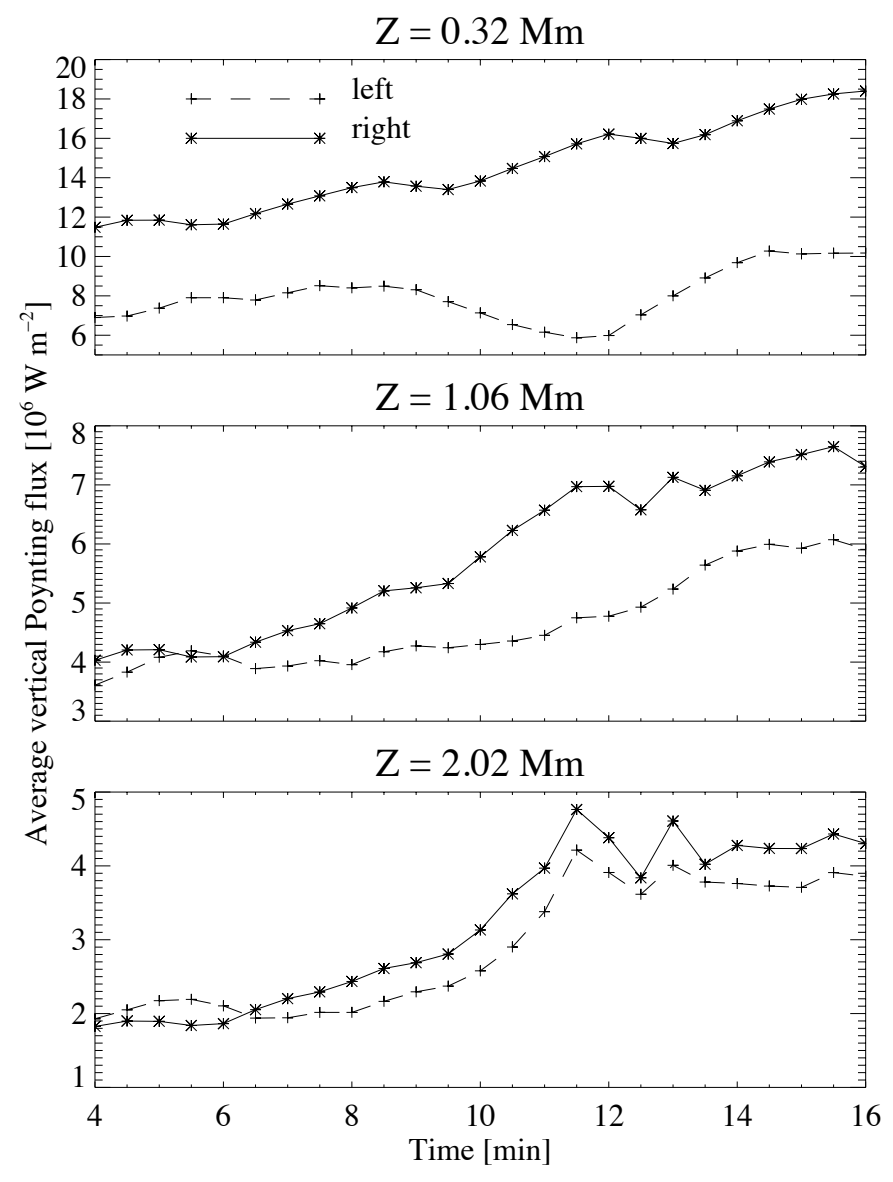

Fig. 10. Temporal evolution of the vertical Poynting flux at the loop footpoints at three different heights from the surface (top panel) to the coronal base (bottom panel). Here we show the averages in horizontal regions around the fieldline indicated in Fig. 9 by the respective boxes. The dashed lines are for the left footpoint, and solid lines are for the right footpoint.

in the coronal part). This underlines that the magnetic disturbance travels from the right coronal base to the left coronal base and triggers there a perturbation, that in the end leads to an increased Poynting flux also on the left side. From the left footpoint at $z=1 \mathrm{Mm}$ we can see that this disturbance can penetrate a bit into the high- $\beta$ region, but cannot reach all the way down into the photosphere (to $z=1 \mathrm{Mm}$ ). This is also because of the strong density stratification.

In conclusion, the time profiles of the Poynting flux at different heights imply that the enhancement at one (right) footpoint near the bottom induces the increase of Poynting flux in higher layers on the same side. This also induces an increase of the Poynting flux on other side of the loop at the coronal base, but not down to the photosphere. As a consequence of the similarly increased Poynting fluxes on both sides at the coronal base, the heat input into the loop is comparably symmetric as already discussed in Sect. 4.2.

Other loops that form later show similar features. A further numerical experiment with increased spatial resolution will have to show if this result can be substantiated. In particular, this will have to investigate to what extent the small-scale evolution of the (inter-)granular magnetic fields can make their way up into the corona and thus alter the spatio-temporal evolution of the Poynting flux in both loop footpoints at the coronal base. 


\section{Summary}

In this paper, we presented a coronal model of an emerging active region driven by a simulation of magnetic flux emergence from the convection zone through the photosphere. The magnetic field expands into the corona, while a pair of simulated spots forms in the photosphere. Ohmic dissipation heats the coronal plasma, while heat conduction along magnetic fieldlines, radiative losses through optically thin radiation, and flows carry away the energy input. The treatment of the full energy balance ensures that the coronal pressure is set self-consistently and allows us to synthesize the EUV emission from the model corona.

Once sufficient magnetic flux was emerged through the surface and the coalescence of small-scale magnetic patches formed large-scale magnetic patches turning into sunspots, the first EUV coronal loops form within minutes. The EUV loop rises upwards, expands significantly in the horizontal direction, and, most importantly, fragments into several individual EUV structures, i.e., the changing heat input produces new strands in a growing envelope.

The energy input is driven by the advection of the magnetic field in the photosphere, i.e., by the horizontal convective motions. Connected by magnetic fieldlines through the corona, the regions of enhanced Poynting flux at one end induce an increase of the Poynting flux at the other end at the coronal base. The upward directed Poynting flux leads to an increased energy input giving rise to the heating of the coronal plasma and the enhancement of the pressure due to the evaporative upflows. The emerging magnetic field hosting the forming loop rises into the ambient magnetic field and currents also build up near the upper part. These contribute to the Ohmic heating in the top part of the loop leading to a nearly constant heat input along the loop.

In its early evolution the coronal loop behaves (at least concerning the energy budget) similarly as a conventional 1D loop model would predict if we would prescribe the energy input. However, in the later stages the loop shows its true 3D nature. The horizontal magnetic expansion and in particular the fragmentation of the EUV emission are a clear indication that a 1D model would not be sufficient to describe a newly forming emerging loop. In the cross-sectional cut perpendicular to the EUV loop, the temperature and the density structure are comparably smooth but not exactly cospatial. This gives rise to the fragmented appearance of the loop in EUV emission with threads (or loop-fragments, or strands) with diameters much smaller than the typical spatial structures in temperature or density.

Our model of the formation and evolution of a EUV coronal loop in an emerging active region sheds new light on our understanding of coronal loop formation. A further analysis of this and more advanced numerical experiments will have to investigate the differences (and similarities) of the evolution of coronal loops seen in different wavelength bands, in particular towards X-rays, and how the forming loops would appear in spectroscopic observations. Of particular interest will be the further investigation of the evolution of the magnetic field structure in relation to the spatial structure of the synthesized coronal emission.

Acknowledgements. We thank M. Schüssler and R. Cameron for discussions and comments that have improved this paper. We gratefully acknowledge the constructive comments by the anonymous referee. This work was supported by the International Max-Planck Research School (IMPRS) on Physical Processes in the Solar System and Beyond. This work was partially funded by the MaxPlanck/ Princeton Center for Plasma Physics. The computations were done at GWDG in Göttingen and SuperMUC. We acknowledge PRACE for awarding us the access to SuperMUC based in Germany at the Leibniz Supercomputing
Centre (LRZ). The visualizations in Fig. 1 and Fig. 6 were done using VAPOR (http: //www .vapor . ucar.edu).

\section{Appendix A: Energy budget along a loop in one dimension}

To investigate the energy budget along the loop structure we made the following assumptions to derive Eq. (1). Under such assumptions, the right-hand side in Eq. (1) basically describes the evolution of the energy along a (field) line that moves with the velocity perpendicular to the line itself.

1. Constant cross section.

$\mathbf{B}$ is invariant along the magnetic tube, which is the same as to say that the loop has a constant cross section. Therefore, the unit vector of the magnetic field, $\mathbf{b}$, satisfies

$$
\nabla \cdot \mathbf{b}=\frac{1}{|\mathbf{B}|} \nabla \cdot \mathbf{B}+\mathbf{B} \cdot \nabla \frac{1}{|\mathbf{B}|}=0 .
$$

2. No compression perpendicular to the loop.

The velocity is decomposed into $u_{\|} \mathbf{b}+u_{\perp} \delta$, with the parallel $u_{\|}=\mathbf{u} \cdot \mathbf{b}$, the perpendicular component $u_{\perp}=\left|\mathbf{u}-u_{\|} \mathbf{b}\right|$, and $\mathbf{b} \cdot \boldsymbol{\delta}=0$. We assume the magnetic tube is not compressed by the flows in the perpendicular direction, which implies $\nabla \cdot\left(u_{\perp} \delta\right)=0$. This assumption is appropriate for a rigid 1D loop model, although it is not the case in our 3D simulation.

3. Heat conduction parallel to the loop.

The heat flux is along the magnetic field, i.e., $\mathbf{q}=q_{\|} \mathbf{b}$. Because $B$ is invariant along the magnetic tube,

$$
\nabla \cdot \mathbf{q}=\mathbf{b} \cdot \nabla q_{\|}+q_{\|} \nabla \cdot \mathbf{b}=\mathbf{b} \cdot \nabla q_{\|} \cdot
$$

These assumptions are consistent with traditional 1D loop modeling. They do not fully hold in the 3D loop we find in our numerical experiment, but are appropriate for the purpose of the comparison made in Sect. 4.

In general, the conservation of thermal energy is written as

$$
\frac{\partial e_{\mathrm{th}}}{\partial t}=-(\mathbf{u} \cdot \nabla) e_{\mathrm{th}}-\frac{\gamma}{\gamma-1} p(\nabla \cdot \mathbf{u})+Q-L-\nabla \cdot \mathbf{q} .
$$

With the above assumptions this energy budget can be rewritten as

$$
\begin{aligned}
\frac{\partial e_{\mathrm{th}}}{\partial t}= & -u_{\|}(\mathbf{b} \cdot \nabla) e_{\mathrm{th}}-u_{\perp}(\boldsymbol{\delta} \cdot \nabla) e_{\mathrm{th}} \\
& -\frac{\gamma}{\gamma-1} p\left[\nabla \cdot\left(u_{\|} \mathbf{b}\right)+\nabla \cdot\left(u_{\perp} \boldsymbol{\delta}\right)\right] \\
& +Q-L-\mathbf{b} \cdot \nabla q_{\|}, \\
\frac{\partial e_{\mathrm{th}}}{\partial t}= & -u_{\|}(\mathbf{b} \cdot \nabla) e_{\mathrm{th}}-u_{\perp}(\boldsymbol{\delta} \cdot \nabla) e_{\mathrm{th}}-\frac{\gamma}{\gamma-1} p(\mathbf{b} \cdot \nabla) u_{\|} \\
& +Q-L-\mathbf{b} \cdot \nabla q_{\|},
\end{aligned}
$$

where the definitions of $Q, L, q_{\|}$are as in Eq. (1). We move the term related to $u_{\perp} \delta$ to the left-hand side of the equation and define

$\left(\frac{\partial e_{\mathrm{th}}}{\partial t}\right)_{s}=\left[\frac{\partial e_{\mathrm{th}}}{\partial t}+u_{\perp}(\boldsymbol{\delta} \cdot \nabla) e_{\mathrm{th}}\right]$.

This can be considered as a type of material derivative.

With this the energy budget reads,

$$
\begin{aligned}
\left(\frac{\partial e_{\mathrm{th}}}{\partial t}\right)_{s}= & -u_{\|}(\mathbf{b} \cdot \nabla) e_{\mathrm{th}}-\frac{\gamma}{\gamma-1} p(\mathbf{b} \cdot \nabla) u_{\|} \\
& +Q-L-\mathbf{b} \cdot \nabla q_{\|},
\end{aligned}
$$

which is just Eq. (1). 


\section{References}

Abbett, W. P., \& Fisher, G. H. 2003, ApJ, 582, 475

Archontis, V., Moreno-Insertis, F., Galsgaard, K., Hood, A., \& O'Shea, E. 2004, A\&A, 426, 1047

Bingert, S., \& Peter, H. 2011, A\&A, 530, A112

Bingert, S., \& Peter, H. 2013, A\&A, 550, A30

Boerner, P., Edwards, C., Lemen, J., et al. 2012, Sol. Phys., 275, 41

Boris, J. P., \& Mariska, J. T. 1982, ApJ, 258, L49

Bourdin, P.-A., Bingert, S., \& Peter, H. 2013, A\&A, 555, A123

Brandenburg, A., \& Dobler, W. 2002, Comput. Phys. Commun., 147, 471

Bray, R. J., Cram, L. E., Durrant, C., \& Loughhead, R. E. 1991, Plasma Loops in the Solar Corona (Cambridge University Press)

Cheung, M. C. M., \& DeRosa, M. L. 2012, ApJ, 757, 147

Cheung, M. C. M., Rempel, M., Title, A. M., \& Schüssler, M. 2010, ApJ, 720, 233

Cook, J. W., Cheng, C.-C., Jacobs, V. L., \& Antiochos, S. K. 1989, ApJ, 338, 1176

Fan, Y. 2001, ApJ, 554, L111

Galsgaard, K., \& Nordlund, Å. 1996, J. Geophys. Res., 101, 13445

Gudiksen, B. V., \& Nordlund, A. 2002, ApJ, 572, L113

Gudiksen, B. V., \& Nordlund, A. 2005a, ApJ, 618, 1020

Gudiksen, B. V., \& Nordlund, A. 2005b, ApJ, 618, 1031

Hansteen, V. H. 1993, ApJ, 402, 741

Hansteen, V. H., Hara, H., De Pontieu, B., \& Carlsson, M. 2010, ApJ, 718, 1070

Heinemann, T., Nordlund, A., Scharmer, G. B., \& Spruit, H. C. 2007, ApJ, 669, 1390

Hurlburt, N. E., Alexander, D., \& Rucklidge, A. M. 2002, ApJ, 577, 993

Klimchuk, J. A. 2006, Sol. Phys., 234, 41

Lionello, R., Winebarger, A. R., Mok, Y., Linker, J. A., \& Mikić, Z. 2013, ApJ, 773,134
Magara, T. 2006, ApJ, 653, 1499

Malanushenko, A., \& Schrijver, C. J. 2013, ApJ, 775, 120

Manchester, IV, W., Gombosi, T., DeZeeuw, D., \& Fan, Y. 2004, ApJ, 610, 588

Martínez-Sykora, J., Hansteen, V., \& Carlsson, M. 2008, ApJ, 679, 871

Martínez-Sykora, J., Hansteen, V., \& Carlsson, M. 2009, ApJ, 702, 129

Müller, D. A. N., Hansteen, V. H., \& Peter, H. 2003, A\&A, 411, 605

Müller, D. A. N., Peter, H., \& Hansteen, V. H. 2004, A\&A, 424, 289

Parker, E. N. 1972, ApJ, 174, 499

Parker, E. N. 1983, ApJ, 264, 642

Parker, E. N. 1988, ApJ, 330, 474

Peter, H. 1999, ApJ, 516, 490

Peter, H. 2007, Adv. Space Res. 39, 1814

Peter, H., \& Bingert, S. 2012, A\&A, 548, A1

Peter, H., \& Judge, P. G. 1999, ApJ, 522, 1148

Peter, H., Gudiksen, B. V., \& Nordlund, A. 2004, ApJ, 617, L85

Peter, H., Gudiksen, B. V., \& Nordlund, A. 2006, ApJ, 638, 1086

Priest, E. R., Heyvaerts, J. F., \& Title, A. M. 2002, ApJ, 576, 533

Reale, F. 2010, Liv. Rev. Sol. Phys., 7, 5

Rempel, M., \& Cheung, M. C. M. 2014, ApJ, submitted [arXiv: 1402 .4703]

Rempel, M., Schüssler, M., Cameron, R. H., \& Knölker, M. 2009, Science, 325, 171

Rosner, R., Tucker, W. H., \& Vaiana, G. S. 1978, ApJ, 220, 643

Spadaro, D., Lanza, A. F., Karpen, J. T., \& Antiochos, S. K. 2006, ApJ, 642, 579

Spitzer, L. 1962, Physics of Fully Ionized Gases, 2nd edn. (New York: Interscience)

van Ballegooijen, A. A., Asgari-Targhi, M., Cranmer, S. R., \& DeLuca, E. E. 2011, ApJ, 736, 3

van Wettum, T., Bingert, S., \& Peter, H. 2013, A\&A, 554, A39

Withbroe, G. L., \& Noyes, R. W. 1977, ARA\&A, 15, 363 